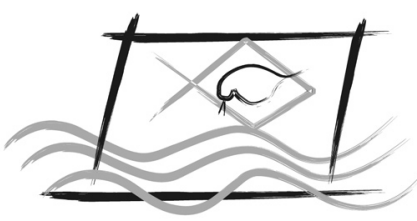

Ecotoxicol. Environ. Contam., v. 8, n. 1, 2013, $41-47$

doi: 10.5132/eec.2013.01.006

ECOTOX - BRASIL

\title{
Respuesta de biomarcadores bioquímicos, morfológicos y comportamentales de la carpa común, Cyprinus carpio, por exposición a muestras ambientales
}

\author{
P.M. CAStaÑÉ ${ }^{1}$, B.L. Eissa ${ }^{1}$ \& N.A. Ossana ${ }^{1,2}$ \\ ${ }^{1}$ Departamento de Ciencias Básicas, Programa de Ecofisiología Aplicada- Instituto de Ecología y Desarrollo Sustentable (PRODEA- \\ INEDES), Universidad Nacional de Luján, CC 221, B6700ZBA -Luján-Argentina. \\ ${ }^{2}$ Consejo Nacional de Investigaciones Científicas y Técnicas (CONICET).
}

(Received November 11, 2011; Accept December 16, 2012)

\begin{abstract}
Resumen
Se caracterizó la calidad ecotoxicológica del agua del río Luján, en 3 sitios de su trayecto con grado dispar de estrés, mediante bioensayos semi-estáticos utilizando la carpa común, Cyprinus carpio. Con un dispositivo y software especial se registró diariamente la ubicación espacial de los peces durante el periodo control (agua potable) y el de exposición (agua del río), y se calculó el Índice de Actividad Relativa ( $I a)$. Se calculó el Factor de Condición ( $F C$ ), y en cerebro se midió la actividad acetilcolinesterásica $(A C h E)$ y el contenido de proteínas totales. La morfología de las branquias se analizó por microscopía electrónica de barrido (MEB). En las muestras de agua se midieron: conductividad, $\mathrm{pH}$, dureza, oxígeno disuelto, nitratos, cloruros, sólidos totales y metales pesados. Se observó en los peces expuestos al agua del río: a) un incremento significativo del $I a$, y de $A C h E$, b) la MEB reveló hipertrofia celular y ligera fusión de las lamelas secundarias. En algunos casos los metales excedieron los límites máximos para protección de la vida acuática. En todas las muestras del río se observó una mezcla compleja de metales pesados y algunos cationes y aniones, que produjeron efectos subletales en los organismos, puestos de manifiesto por la respuesta de los biomarcadores determinados.
\end{abstract}

Palabras claves:Río Luján, Cyprinus carpio, Acetilcolinesterasa cerebral, Índice Actividad Natatoria, Microscopía Electrónica de Barrido.

\section{Biochemical, morphological and behavioural biomarkers responses of common carp, Cyprinus carpio, exposed to environmental samples}

\begin{abstract}
Ecotoxicological quality from Luján river water in three sites with different degree of stress, through semi-static bioassay using the commom carp Cyprinus carpio as test organism was characterized. A computer based-data acquisition system daily registered the spatial location of the fishes during the control period (freshwater) and the exposition period (river water), and the Relative Activity Index was calculated $(A i)$. The Condition Factor $(C F)$ was calculated and $C F$ and Acetilcholinesterase activity $(A C h E)$ and total protein content were measured in brain. The morphology of the gills was analyzed by scanning electron microscopy (SEM). In sampling water conductivity, $\mathrm{pH}$, hardness, dissolved oxygen, nitrates, chlorides, total solids and heavy metals were measured. It was observed in individuals exposed to environmental samples: a) $A i$ and $A C h E$ increased significatively, b) SEM revealed cellular hypertrophy and slight melting of secondary lamellae in individuals. In some cases, metals exceeded the maximum permitted quantities for protection of aquatic life. In all river samples a complex mixture of heavy metals and some anions and cations was observed, which produced sublethal effects in the organisms, revealed by the biomarkers responses.
\end{abstract}

Keywords: Lujan River, Cyprinus carpio, brain acetilcholinesterase, Swimming Activity Index, Scanning Electron Microscopy.

*Corresponding author: Patricia M. Castañé; e-mail: prodea@mail.unlu.edu.ar 


\section{INTRODUCCIÓN}

La caracterización de la calidad toxicológica de los cuerpos de agua continentales puede abordarse con el auxilio de diversas técnicas ya sea evaluando parámetros fisicoquímicos de las muestras ambientales, y también mediante la determinación en organismos acuáticos de biomarcadores de distinta naturaleza (bioquímicos, morfológicos). Más recientemente se han incorporado métodos que contribuyen al mismo propósito mediante el análisis de alteraciones etológicas.

Cada una de ellas brinda información referente a un aspecto particular de dicha caracterización; cuando son aplicadas en forma conjunta permiten acceder a una información integrada de la calidad ecotoxicológica de las muestras consideradas y también permiten conocer sus cambios espaciales y temporales así como establecer relaciones causales de los cambios que pueden advertirse.

El Río Luján pertenece a la denominada Pampa húmeda y por sus características geológicas a la llanura Chaco Pampeana. Es una de las tres cuencas periurbanas del NE de la Provincia de Buenos Aires; las otras dos son la de los ríos Reconquista y Matanza-Riachuelo. Corre en dirección SO-NO a lo largo de $128 \mathrm{~km}$, y desemboca en el Estuario del Plata. Su caudal proviene principalmente del drenaje de los excedentes hídricos provenientes de las importantes precipitaciones que a lo largo de todo el año caen en su amplia cuenca de casi 2700 $\mathrm{km}^{2}$ y de los afloramientos freáticos (Sanchez Caro, 2010) aguas debajo de sus nacientes. A la altura de la localidad de Jáuregui, el río se halla embalsado por una pequeña represa hidroeléctrica privada; otra obra similar, de menor magnitud y fines recreativos, se encuentra en la ciudad de Luján. El río atraviesa regiones rurales, periurbanas y urbanas de 13 municipios.

La zona ha sido y es utilizada para explotación agrícologanadera; sin embargo, con el crecimiento de las ciudades se ha incrementado también la actividad industrial, especialmente en la cuenca inferior que está constituida por los arroyos y tierras que drenan en el tramo del río más cercano a la desembocadura (Giorgi, 2000).

El objetivo del presente estudio ha sido determinar mediante bioensayos de laboratorio, los efectos de muestras del agua del río Luján, en sitios de su trayecto con grado dispar de estrés, sobre biomarcadores bioquímicos, morfológicos y comportamentales de la carpa común, Cyprinus carpio.

\section{MATERIALES Y MÉTODOS}

El estudio se llevó a cabo en invierno y primavera de 2005, y en verano de 2006. Las muestras provinieron de tres sitios: 1) M. J. García; zona de bajo impacto antrópico que fue considerada como "sitio control"; 2) Jáuregui, área industrial y 3) Cruce del río con Ruta 6, localizado a $12 \mathrm{Km}$ después de atravesar el ejido urbano de la ciudad de Luján (Fig. 1).

Las muestras fueron tomadas de la superficie del cuerpo de agua y transportadas al laboratorio refrigeradas y acidificadas

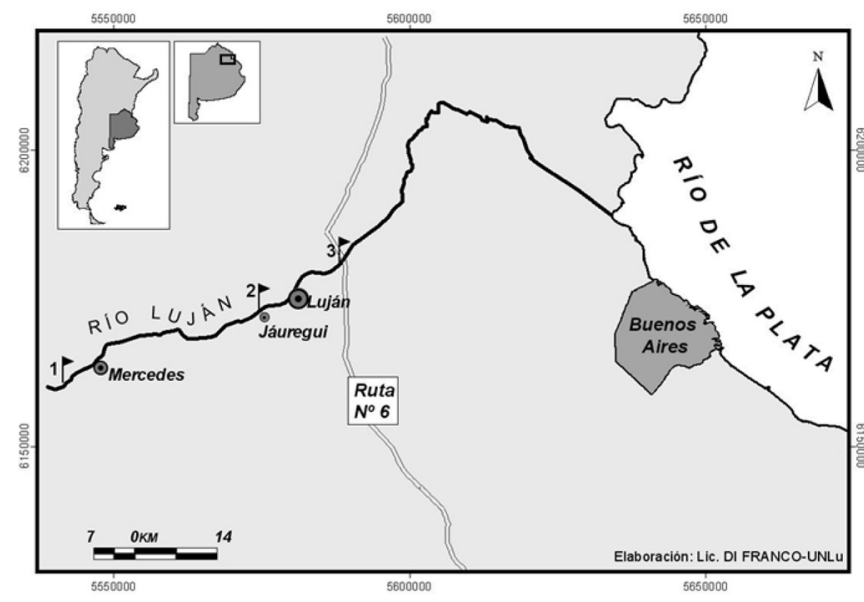

Figura 1: Mapa de la Cuenca del Río Luján y ubicación de las estaciones de muestreo. 1) M. J. García; 2) Jáuregui; 3) Cruce del río con Ruta 6.

$(\mathrm{pH}<2)$ (para la determinación de metales pesados). Sobre alícuotas de las mismas se determinó: $\mathrm{pH}$, conductividad, dureza, sólidos totales, oxígeno disuelto (OD), nitrato, cloruros y metales pesados (APHA, AWWA, WEF, 2005).

Se utilizaron 34 ejemplares juveniles de Cyprinus carpio (peso corporal, 3.3-3.7 g; largo total, 6.0-6.6 cm) provenientes de un criadero comercial, sin exposición previa a contaminantes. Los peces fueron aclimatados en agua potable (AP) de la ciudad de Luján (Buenos Aires), a temperatura $\left(21 \pm 2{ }^{\circ} \mathrm{C}\right)$ y fotoperíodo $(12: 12 \mathrm{~L}: \mathrm{O})$ constantes, con flujo cerrado y continuo; la aireación fue permanente.

Los bioensayos fueron de tipo semi-estático y se llevaron a cabo en acuarios de vidrio de 20-24 L de capacidad. Con un dispositivo y software especial se registró diariamente la ubicación espacial de los peces durante el ensayo; el dispositivo utilizado se describió en Eissa et al. (2006a). Se colocó un animal en cada acuario con aireación permanente; el flujo fue continuo y cerrado, a una velocidad de $20-25 \mathrm{~mL}$ $\mathrm{min}^{-1}$, regulado mediante una bomba peristáltica multicanal Cole Palmer.

Los ensayos tuvieron dos periodos de cuatro días de duración cada uno. En el primero (Período Control; C) los acuarios contuvieron agua potable (AP) y en el segundo (Período Experimental) ese medio fue reemplazado por las muestras del agua del río; de esta forma cada animal fue control de sí mismo. Se determinó periódicamente $\mathrm{pH}, \mathrm{OD}$, dureza y conductividad en el agua de los medios de incubación de los bioensayos.

Cabe señalar que en todos los ensayos se corrió en paralelo un Control Permanente [CoP] "externo" en el cual un pez permaneció en forma continua durante 8 días en AP circulante.

Los muestreos focales diarios, permitieron determinar la Actividad Natatoria Total expresada como el Índice de Actividad Relativa (Ia) (Eissa, 2009; Eissa et al., 2006a). El mismo se calculó dividiendo el número promedio de movimientos totales registrados diariamente durante 4 horas en cada período $(\mathrm{C}, \mathrm{E})$ por el número total de movimientos 
registrados en un día particular $i$ del período. Cuando $I a=1$ indica que no se produjeron cambios en la actividad natatoria total, mientras que si $I a>1$ indica disminución en la actividad natatoria, lo contrario corresponde cuando $I a<1$.

Al finalizar cada período, los peces fueron removidos, anestesiados por inmersión en agua potable con hielo, pesados (precisión $\pm 0.1 \mathrm{~g}$ ) y medidos (precisión $\pm 0.1 \mathrm{~cm}$ ). Con estos valores se calculó el Factor de Condición $(F C=($ peso $\mathrm{x} 100)$ largo total ${ }^{-3}$ ) (Sloof et al., 1983).

Luego se realizó la disección del cerebro para determinar el contenido de proteínas tisular (método de Lowry et al., 1951) y la actividad de la acetilcolinesterasa ( $A C h E$, método de Ellman et al., 1961). Todas las mediciones se realizaron por triplicado.

Para la microscopía de las branquias, luego de su extracción, las mismas se lavaron varias veces con buffer fosfato de sodio al $1 \%$ (pH 7.2), y se fijaron en Glutaraldehído $2.5 \%$ en buffer fosfato durante $48 \mathrm{hs}$. Luego se procedió a la deshidratación en series de alcohol y acetona. Finalmente, se desecaron con la técnica del Punto Crítico y se revistieron con oro-paladio evaporado. Se tomaron registros fotográficos de las muestras entre 10 y $15 \mathrm{kv}$ en un Microscopio Electrónico de Barrido (MEB) JEOL SLM 100 (servicio de Microscopía Electrónica de Barrido, Facultad de Ciencias Naturales y Museo, UNLP) (Ferrari et al., 2009; Ossana et al., 2010).

Para evaluar las diferencias en la actividad natatoria de los grupos se utilizó ANOVA de un factor para muestras pareadas. El supuesto de normalidad se probó con el test de Kolmogorov-Smirnov y el de homogeneidad de varianzas con el test de Bartlett. En los casos donde no se cumplía homocedacia, se realizó el test no paramétrico de Friedman para medidas repetidas y el de Dunn (Siegel \& Castellan, 1995). Las diferencias entre los grupos para $A C h E$ y $F C$ se analizaron mediante el test no paramétrico de Kruskal Wallis y el de Dunn (Zar, 2010).

Se realizó un PCA (Análisis de Componentes Principales) con los parámetros fisicoquímicos donde se incluyeron los metales pesados, cloruros y nitratos. Previamente se probó la normalidad con el test de Kolmogorov-Smirnov y todos los datos fueron estandarizados. Se realizó una correlación de Pearson entre la Actividad Natatoria y los Factores del PCA junto con un análisis estadístico (test de $t$ ).

Los análisis estadísticos se realizaron con el programa InfoStat para la actividad natatoria, AChE y FC, y se utilizó el programa Statistica para Windows para el PCA y la correlación de Pearson.

\section{RESULTADOS}

En la Tabla 1 se muestran los resultados de los análisis fisicoquímicos del agua de cada una de las muestras ambientales $\mathrm{y}$ de los medios de incubación de los bioensayos.

En la Tabla 2 se indican los $I a$ para cada grupo experimental en cada sitio. En la Tabla 3 se presentan los resultados de $A C h E$ y $F C$.

Los primeros 4 Factores del PCA tuvieron una varianza acumulativa del $85.61 \%$ de la varianza total. Para el primer Factor resultaron significativas las variables: $\mathrm{Pb}(-0.8), \mathrm{Cr}(-0.94)$ y $\mathrm{Mn}$ $(-0.7)$; para el segundo Factor: $\mathrm{Cu}(-0.81)$ y para el tercer Factor: $\mathrm{Cl}(-0.7)$ y $\mathrm{Zn}(-0.81)$. De las correlaciones entre los Factores del PCA y el $I a$, sólo resultó estadísticamente significativa $(\mathrm{p}=0.0049)$ entre el $I a$ y el Factor 3 (Fig. 2), positiva con un coeficiente de correlación de Pearson (r) de 0.707.

En la Figura 3 se presentan las imágenes de las branquias de los peces controles y expuestos a muestras del río.

Tabla 1. Parámetros fisicoquímicos de los controles (agua potable) y de las muestras de agua del río Luján. *Indican mediciones realizadas sobre muestras de agua del río y durante el transcurso de los bioensayos; se indican los límites máximos-mínimos registrados durante el período estudiado. Entre paréntesis: número de mediciones; ND: no detectado; NM: no medido. \# Niveles Guía según Ley Nacional de Residuos peligrosos ํㅜ 24051, Argentina.

\begin{tabular}{|c|c|c|c|c|c|c|c|c|c|c|c|c|}
\hline & & $\begin{array}{l}\text { Control } \\
\text { (AP) }\end{array}$ & & M.J.García & & & Jáuregui & & & Ruta 6 & & $\begin{array}{c}\text { Niveles } \\
\text { Guía\# }\end{array}$ \\
\hline Parámetro & Unidades & & $\begin{array}{c}\text { Invierno } \\
05\end{array}$ & $\begin{array}{c}\text { Primavera } \\
05 \\
\end{array}$ & $\begin{array}{c}\text { Verano } \\
06\end{array}$ & $\begin{array}{c}\text { Invierno } \\
05\end{array}$ & $\begin{array}{c}\text { Primavera } \\
05\end{array}$ & $\begin{array}{c}\text { Verano } \\
06\end{array}$ & $\begin{array}{c}\text { Invierno } \\
05\end{array}$ & $\begin{array}{c}\text { Primavera } \\
05 \\
\end{array}$ & $\begin{array}{c}\text { Verano } \\
06\end{array}$ & $\mu \mathrm{g} \mathrm{L}^{-1}$ \\
\hline $\mathrm{pH}^{*}$ & & $\begin{array}{c}8.3-9.4 \\
(72)\end{array}$ & $\begin{array}{l}8.2-9.0 \\
(24)\end{array}$ & $\begin{array}{l}7.9-9.1 \\
(8)\end{array}$ & $\begin{array}{c}8.7-8.9 \\
(8)\end{array}$ & $\begin{array}{c}7.7-9.5 \\
(24)\end{array}$ & $\begin{array}{c}8.5-8.7 \\
(8)\end{array}$ & $\begin{array}{l}8.8-9.0 \\
(8)\end{array}$ & $\begin{array}{c}7.7-9.5 \\
(24)\end{array}$ & NM & $\begin{array}{c}8.5-8.7 \\
(8)\end{array}$ & \\
\hline OD* & $\mathrm{mg} \mathrm{O}_{2} \mathrm{~L}^{-1}$ & $\begin{array}{c}6.8-8.6 \\
(32)\end{array}$ & $\begin{array}{c}7.0-7.6 \\
(4)\end{array}$ & $\begin{array}{l}7.0-7.4 \\
(4)\end{array}$ & $\begin{array}{c}7.1-7.3 \\
(4)\end{array}$ & $\begin{array}{c}6.6-7.2 \\
(4)\end{array}$ & $\begin{array}{c}7.9-8.3 \\
(4)\end{array}$ & $\begin{array}{c}7.0-7.3 \\
(4)\end{array}$ & $\begin{array}{c}6.6-7.2 \\
(4)\end{array}$ & NM & $\begin{array}{c}7.5-8.3 \\
(4)\end{array}$ & \\
\hline Dureza* & $\mathrm{mg} \mathrm{CaCO}_{3} \mathrm{~L}^{-1}$ & $\begin{array}{c}75.9-82.3 \\
(32)\end{array}$ & $\begin{array}{c}215.6-217.4 \\
\text { (4) }\end{array}$ & $\begin{array}{c}119.8-164.0 \\
\text { (4) }\end{array}$ & $\begin{array}{c}119.8-164.0 \\
\text { (4) }\end{array}$ & $\begin{array}{c}262.7-264.5 \\
\text { (4) }\end{array}$ & $\begin{array}{c}152.7-154.3 \\
\text { (4) }\end{array}$ & $\begin{array}{l}92.8-95.8 \\
(4)\end{array}$ & $\begin{array}{c}162.7-164.5 \\
\text { (4) }\end{array}$ & NM & $\begin{array}{c}152.7-154.3 \\
\text { (4) }\end{array}$ & \\
\hline $\begin{array}{l}\text { Conducti- } \\
\text { vidad* }\end{array}$ & $\mathrm{mS} \mathrm{cm}{ }^{-1}$ & $\begin{array}{l}0.72-0.84 \\
(32)\end{array}$ & $\begin{array}{c}1.88-1.92 \\
(4)\end{array}$ & $\begin{array}{c}1.15-1.36 \\
(4)\end{array}$ & $\begin{array}{c}1.10-1.13 \\
(4)\end{array}$ & $\begin{array}{c}1.53-1.57 \\
(4)\end{array}$ & $\begin{array}{c}1.45-1.51 \\
(4)\end{array}$ & $\begin{array}{l}0.89-0.90 \\
(4)\end{array}$ & $1.53-1.57$ (4) & NM & $\begin{array}{l}0.94-0.96 \\
\text { (4) }\end{array}$ & \\
\hline Cloruros & $\mathrm{mg} \mathrm{Cl}^{-} \mathrm{L}^{-1}$ & NM & 371 & 1 & 206.4 & 268 & 379.7 & 46.1 & 285 & $\mathrm{NM}$ & 53.4 & \\
\hline $\begin{array}{l}\text { Sólidos } \\
\text { Totales }\end{array}$ & & NM & 2152 & 2580 & 1210 & 1870 & 2130 & 600 & 1939 & $\mathrm{NM}$ & NM & \\
\hline Nitratos & $\mathrm{mg} \mathrm{NO}_{3}-\mathrm{N} \mathrm{L}^{-1}$ & NM & 1.4 & 2 & 1.45 & 0.7 & 12 & 1.97 & 0.6 & $\mathrm{NM}$ & NM & \\
\hline Hierro & $\mathrm{mg} \mathrm{L}^{-1}$ & NM & 1.06 & $<0.01$ & 0.01 & 0.3 & $<0.01$ & 0.05 & 0.03 & $\mathrm{NM}$ & $\mathrm{NM}$ & \\
\hline Manganeso & $\mathrm{mg} \mathrm{L}^{-1}$ & $\mathrm{NM}$ & 250 & 240 & $<5$ & 70 & 25 & 18 & 10 & NM & 42 & 100 \\
\hline Plomo & $\mathrm{mg} \mathrm{L}^{-1}$ & ND & $<30$ & 31 & $<20$ & 20 & 22 & $<20$ & 30 & NM & $<20$ & 1 \\
\hline Cobre & $m g L^{-1}$ & NM & 10 & 9 & $<10$ & 4 & 7 & $<10$ & 5 & NM & $<10$ & 2 \\
\hline Zinc & $\mathrm{mg} \mathrm{L}^{-1}$ & NM & 30 & $<5$ & 50 & 7 & 17 & $<10$ & 8 & NM & NM & 30 \\
\hline Cadmio & $m g \mathrm{~L}^{-1}$ & ND & 3 & 3 & $<2$ & $<2$ & $<2$ & $<2$ & 2 & NM & $<20$ & 0.2 \\
\hline Cromo & $\mathrm{mg} \mathrm{L}^{-1}$ & ND & 20 & 20 & 6 & $<20$ & $<20$ & $<6$ & $<20$ & NM & $<6$ & 2 \\
\hline
\end{tabular}


Tabla 2. Índice de Actividad (Ia) de juveniles de Cyprinus carpio expuestos a muestras del río Luján; Controles en agua potable. Datos como medias \pm ESM. Los asteriscos indican diferencias significativas con el control $\mathrm{p}<0.05$. NM: no medido.

\begin{tabular}{lcccccc}
\hline & \multicolumn{2}{c}{ Invierno 2005 } & \multicolumn{2}{c}{ Primavera 2005 } & \multicolumn{2}{c}{ Verano 2006 } \\
\cline { 2 - 7 } & Control & Exposición & Control & Exposición & Control & Exposición \\
\hline M.J.García & $1.14 \pm 0.22$ & $1.07 \pm 0.14$ & $1.00 \pm 0.02$ & $3.33 \pm 1.08^{*}$ & $1.01 \pm 0.72$ & $1.07 \pm 0.12$ \\
Jáuregui & $1.01 \pm 0.05$ & $1.06 \pm 0.13^{*}$ & $1.02 \pm 0.03$ & $1.11 \pm 0.08$ & $1.03 \pm 0.11$ & $1.07 \pm 0.14$ \\
Ruta 6 & $1.02 \pm 0.06$ & $1.42 \pm 0.47^{*}$ & $\mathrm{NM}$ & $\mathrm{NM}$ & $1.02 \pm 0.08$ & $2.04 \pm 0.79^{*}$ \\
\hline
\end{tabular}

Tabla 3. Actividad acetilcolinesterasa cerebral (AChE, $\mathrm{mmol} \mathrm{min}^{-1} \mathrm{~g}$ proteína $\left.^{-1}\right)$, proteínas tisulares de cerebro $\left(\mathrm{mg}\right.$ proteina $\mathrm{g}$ peso fresco $\left.{ }^{-1}\right) \mathrm{y}$ Factor de condición (FC) de juveniles de Cyprinus carpio. N: número de animales. Datos como medias \pm ESM. * Diferencias significativas entre grupos con el mismo símbolo $(\mathrm{p}<0.05)$.

\begin{tabular}{|c|c|c|c|c|c|}
\hline Sitio & $\begin{array}{l}\text { Estación } \\
\text { del año }\end{array}$ & & 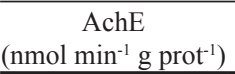 & $\begin{array}{l}\text { Proteína cerebro } \\
\left(\mathrm{mg} \mathrm{g}_{\text {g ejido }}\right)^{-1}\end{array}$ & $\mathrm{FC}$ \\
\hline Control & & 6 & $7206+689 *$ & $46.94+3.13$ & 8 \\
\hline cía & a & 6 & $69+1153$ & & 125 \\
\hline & Ver: & 4 & $96.47 \pm 18.48$ & $100.31 \pm 9.23^{*}$ & $1.31 \pm 0.03$ \\
\hline Jauregui & Primavera & 3 & $99.59 \pm 18.70$ & $79.21 \pm 3.78$ & $1.14 \pm 0.05$ \\
\hline Jauregui & Verano & 4 & $137.44 \pm 1$ & $43.59 \pm$ & $1.26 \pm 0.03$ \\
\hline uregui & Invierno & 3 & $118.43 \pm$ & $45.99 \pm 3$ & $1.23 \pm 0.04$ \\
\hline & Primavera & 4 & $116.12 \pm 4.05$ & $47.92 \pm 4$ & $1.30 \pm 0.21$ \\
\hline Ruta 6 & Verano & 4 & $128.09 \pm 17.02$ & $39.31 \pm 4.11^{*}$ & $1.04 \pm 0.03$ \\
\hline
\end{tabular}



Figura 2 - Correlación entre la Actividad Natatoria $(I a)$ y el Factor 3 del PCA.

\section{DISCUSIÓN}

La degradación de la calidad del ecosistema acuático resulta en alteraciones de sus propiedades físico-químicas y tiene repercusión sobre toda la biota. Algunas sustancias pueden ser muy tóxicas en forma aislada pero su toxicidad se puede ver mitigada o aumentada originando acciones antagónicas o sinérgicas cuando se encuentra formando parte de un efluente debido a la interacción con otros compuestos (Grothe et al., 1996).

En cuanto a los parámetros fisicoquímicos analizados se detectó que el $\mathrm{Mn}, \mathrm{Pb}, \mathrm{Cu}, \mathrm{Zn}, \mathrm{Cr}$ y Cd excedieron los límites máximos para protección de la vida acuática aceptados por la Legislación Nacional.

En los bioensayos conductuales el valor del $I a$, en el período Control, fue estable oscilando en torno a 1. En tanto que en el caso de los peces expuestos durante 4 días al agua proveniente del Río Luján se observó una elevación significativa del mismo en las muestras procedentes de Ruta 6, y en los otros sitios de muestreo en una de las estaciones del año estudiada (Primavera en el caso de M.J.García e Invierno en el caso de Jauregui) (Tabla 2). Dichas respuestas, fueron indicativas de una depresión de la actividad de los peces. Resultados similares a los nuestros observaron Gerhardt et al. (2002) estudiando el efecto sobre peces y crustáceos de una serie de diluciones de agua, proveniente de una planta de tratamiento de aguas residuales municipales, observando cambios en la actividad natatoria y en los movimientos ventilatorios de los peces.

En el caso de los CoP se apreció que los Ia presentaron un valor próximo a 1 , lo que es indicador de que no hubo cambios significativos en la actividad natatoria espontánea durante el periodo experimental ( 8 días). Estos valores fueron similares a los observados en el periodo control (Tabla 2) por este motivo no fueron incluidos en dicha Tabla.

Según los trabajos de Chuiko y col. (2003, 2000) la carpa común (Cyprinus carpio) posee únicamente AChE. En nuestro trabajo se observó un aumento de la actividad de esta enzima en las tres estaciones estudiadas respecto del control en AP, que resultó estadísticamente significativo en Jáuregui verano (Tabla 3). Es posible que algunos tóxicos o condiciones ambientales de las muestras de río sean los responsables de dichos aumentos, como ser, herbicidas, algunos metales pesados, elevadas concentraciones de $\mathrm{NH}_{4}$ y/o reducido oxígeno disuelto (Rodríguez Kaizer et al., 2009; Liu et al., 2009).

No se observaron diferencias significativas entre los peces expuestos al agua de río y los controles en AP tanto para el contenido tisular de las proteínas de cerebro como para el FC.

La correlación entre los parámetros fisicoquímicos, y la actividad natatoria fue significativa y positiva para el $\mathrm{Zn}$ y los cloruros, (parámetros significativos del Factor 3). Esto indicaría que a medida que aumentan estos factores aumenta el índice $I a$, el cual es inversamente proporcional a la actividad de nado de los peces. La concentración en el medio de estos iones estaría relacionado con la depresión en la actividad de nado de los peces expuestos.

Las branquias son órganos blanco críticos frente a la exposición a tóxicos debido a su extensa área en contacto 


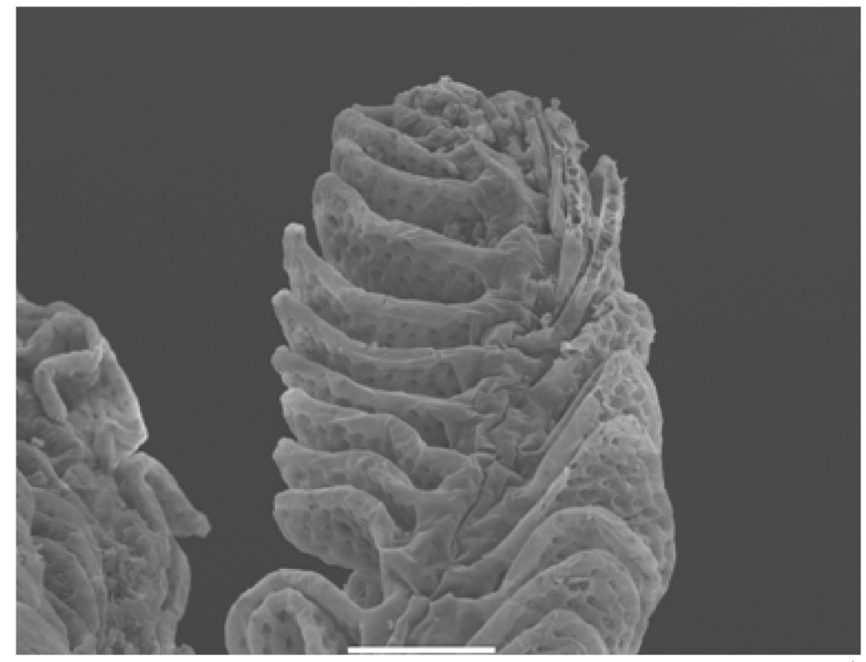

A

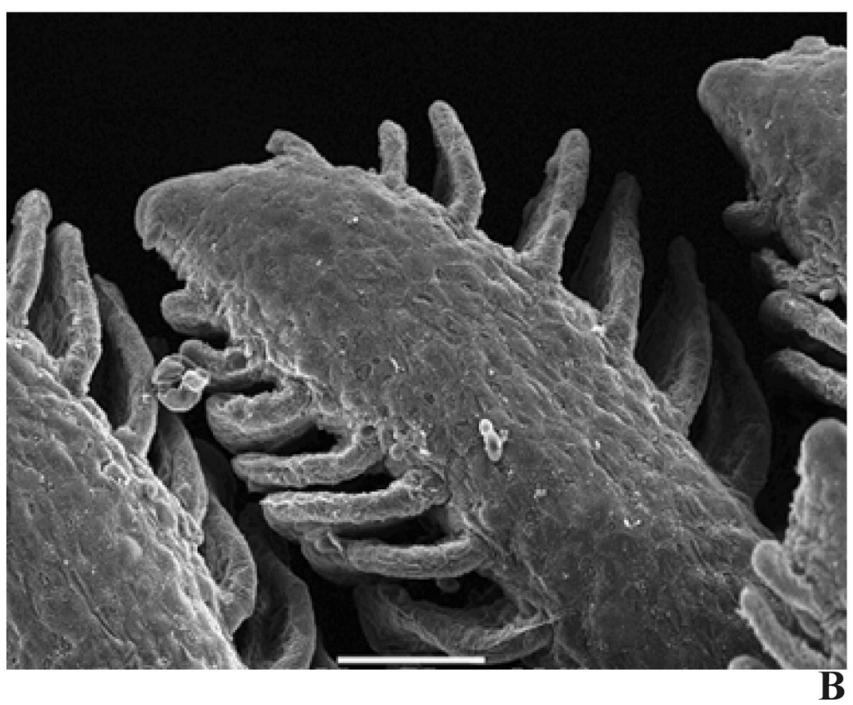

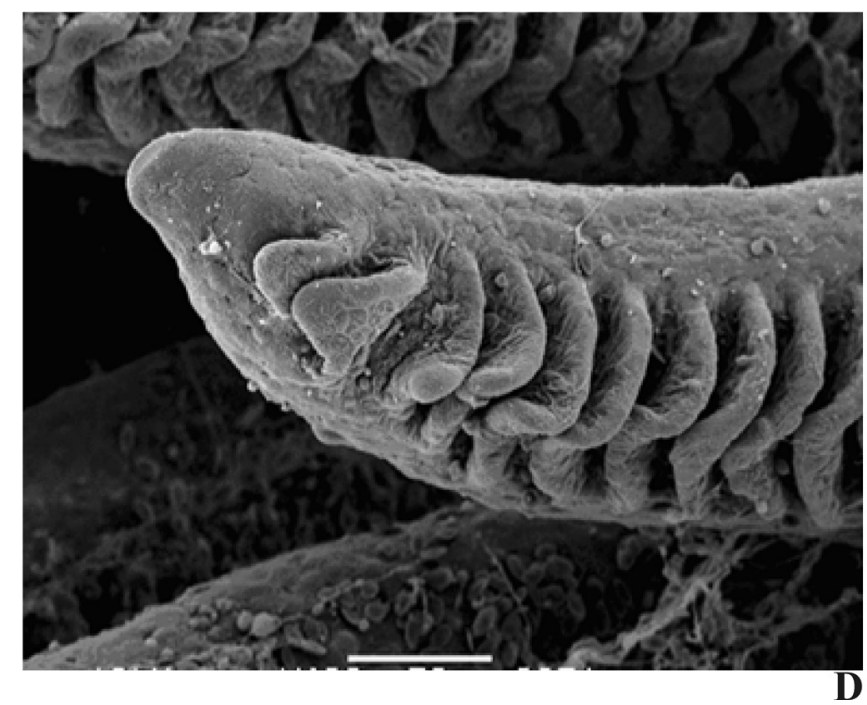

Figura 3 - Imágenes al MEB de las lamelas secundarias de Cyprinus carpio: A, en agua potable; B, expuesto a agua proveniente de M.J. García; C, expuesto a agua proveniente de Jáuregui; D, expuesto a agua proveniente de Ruta 6. Aumento: 430X; barras: $50 \mu \mathrm{m}$.

con el medio externo y al reducido espesor de las membranas que se interponen entre el medio interno y el externo. En la Figura 3.a se mostró detalle de la lamela secundaria de las branquias de un individuo mantenido en agua potable, con sus límites celulares bien definidos y sin signos de hipertrofia e hiperplasia. Las mismas se observaron como finas solapas semicirculares dispuestas de forma bilateral y simétrica con respecto a las lamelas primarias. Las imágenes de animales expuestos a agua de M. J. García (Figura 3.b) son comparables con las de animales control.

En la microscopía de branquias de los animales expuestos al agua proveniente de Jauregui (Figura 3.c) se observó una leve hipertrofia celular caracterizada por una reducción en la visualización de los límites celulares tal vez debido a una edematización. Esta misma patología se apreció en las branquias de animales expuestos a agua de Ruta 6 (Figura 3.d). También se observó deformación de las lamelas primarias y secundarias mostrando a su vez una leve fusión de las mismas. Diversos autores han informado alteraciones estructurales y cambios morfopatológicos en peces frente a la exposición a contaminantes.
Ossana (2011) observó en branquias de larvas de Lithobates catesbeianus expuestas a muestras ambientales del sitio Ruta 6 alteraciones similares; abundante secreción de mucus, desestructuración, desbalance osmótico, colapso de los filamentos branquiales y edematización. En algunas muestras también se observó colonización de bacilos y cocos.

Thophon et al. (2004) observaron edema de células epiteliales, aneurisma en lamelas secundarias, ruptura de células mucosas, hipertrofia e hiperplasia de células epiteliales y de cloruro en Lates calcarifer expuestos a Cadmio. Domitrovic (1997) observó hiperplasia e hipertrofia epitelial, fusión de laminillas y de filamentos. Brunelli et al. (2011) observaron en el teleósteo, Thalassoma pavo L, expuesto a Cadmio alteraciones histopatológicas. En este trabajo a concentraciones intermedias se observó ausencia de microvellosidades particularmente en la porción proximal y a lo largo de los márgenes de las lamelas; a mayores concentraciones las branquias mostraron una apariencia irregular y la superficie del epitelio estaba replegado en varios puntos, las lamelas secundarias se afinaron y se replegaron en la porción distal. 
El epitelio de las branquias es el sitio de intercambio de gases, regulación iónica, balance ácido-base y excreción de nitrógeno, los tres últimos procesos son controlados por fenómenos de transporte pasivo y activo de varios solutos a través del epitelio de las branquias a nivel celular, las que influyen en los sistemas de regulación iónica (Evans et al., 2005). Es evidente que el estado de las mismas principalmente en lo referente al cambio morfológico de las lamelas secundarias debe disminuir la eficiencia respiratoria y el grado de su deterioro condicionará la reversibilidad de las alteraciones.

La mezcla de contaminantes presente en el río puede provocar en los peces sofocamiento debido a precipitados de mucoproteínas sobre el epitelio branquial, interfiriendo en procesos fisiológicos fundamentales radicados en ese órgano lo cual a su vez puede secundariamente interferir en la performance de nado.

Las alteraciones observadas pueden estar relacionadas con las concentraciones de los aniones y cationes, así como la mezcla compleja de metales pesados encontrados en las muestras de agua del río. Como se ha mencionado anteriormente se determinó una correlación entre algunos parámetros fisicoquímicos y el Índice de Actividad Natatoria. Cabe señalar que las concentraciones de esos compuestos no alcanzaron niveles de letalidad ya que en ningún caso se registró mortalidad de los peces. No obstante, se detectaron efectos subletales puestos de manifiesto por la respuesta de los biomarcadores, como ser, aumento en la actividad de Acetilcolinesterasa y depresión en la actividad natatoria.

La actividad de la AChE cerebral es extremadamente importante en muchos procesos fisiológicos, como ser, evasión de predadores, localización de la presa y orientación hacia la comida. La activación o inhibición de la esta enzima puede influir en procesos colinérgicos de neurotransmisión y promover efectos no deseados (Moraes et al., 2007). Ossana (2011) encontró respuestas similares exponiendo larvas de $L$. catesbeianus a muestras del mismo sitio aquí analizado, con aumentos significativos en la actividad de la AChE cerebral en los muestreos realizados en primavera y verano. En este caso también se encontraron elevados niveles de amonio así como $\mathrm{Zn}, \mathrm{Cu}$ y $\mathrm{Cr}$ y bajos niveles de oxígeno. Rodriguez Kaizer et al. (2009) mostró que la actividad de esta enzima en el cerebro de Rhamdia quelen incrementó un 39\% cuando fue expuesta $24 \mathrm{~h}$ a bajos niveles de oxígeno y altas concentraciones de amonio, una condición comparable a la encontrada en este río. Nuestros resultados indicaron que dado que las características fisicoquímicas y biológicas de los ambientes acuáticos son extremadamente diversas y variables, la respuesta individual de un solo biomarcador no se puede considerar suficiente para brindar diagnosis completa de los efectos de la polución. Sin embargo, el empleo de una batería de biomarcadores en forma conjunta con otras mediciones químicas y biológicas permitiría alcanzar dicho objetivo (Cajaraville et al., 2000).

\section{BIBLIOGRAFIA}

APHA, AWWA \& WEF, 2005, Standard Methods: for the examination of water and wastewater. 19 th Edition.
BRUNELLI, E., MAUCERI, A., MAISANO, M., BERNABO, I., GIANNETTO, A., DE DOMENICO, E., CORAPI, B., TRIPEPI, S. \& FASULO, S., 2011, Ultrastructural and immunohistochemical investigation on the gills of the teleost, Thalassoma pavo L., exposed to cadmium. Acta Histochem., 113:201-13. http://dx.doi.org/10.1016/j.acthis.2009.10.002

CAJARAVILLE, M.P., BEBIANNO, M.J., BLASCO, J., PORTE, C., SARASQUETE, C. \& VIARENGO, A., 2000, The use of biomarkers to assess the impact of pollution in coastal environments of the Iberian Peninsula: a practical approach. Sci. Total Environ., 247: 295-311. http://dx.doi.org/10.1016/S00489697(99)00499-4

CHUIKO, G.M., PODOGORNAYA, V.A. \& ZHELNIN Y.Y. (2003) Acetylcholinesterase and butyrylcholinesterase activities in brain and plasma of freshwater teleosts: cross-species and cross-family differences. Comp Biochem Physiol. B , 135:55-61. http://dx.doi. org/10.1016/S1096-4959(03)00048-4

CHUIKO, G.M. (2000) Comparative study of acetylcholinesterase and butyrylcholinesterase in brain and serum of several freshwater fish: specific activities and in vitro inhibition by DDVP, an organophosphorus pesticide. Comp. Biochem. Physiol. C, 127, 233-242. http://dx.doi.org/10.1016/S0742-8413(00)00150-X

DOMITROVIC, H.A., 1997, Toxicidad y respuesta histopatológica en Aequidens portalegrensis (PISCES, Cichlidae) expuestos a sulfato de cobre en ensayos de toxicidad aguda y en ensayos subletales. Reunión de Comunicaciones Científicas y Tecnológicas de la Universidad Nacional del Nordeste, Tomo II: 25-28.

EISSA, B.L., 2009, Biomarcadores comportamentales, fisiológicos y morfológicos de exposición al Cadmio en peces pampeanos. Tesis Doctoral, Facultad de Ciencias Exactas y Naturales, Universidad de Buenos Aires, 200 pp.

EISSA, B.L., FERRARI, L., OSSANA, N.A. \& SALIBIÁN, A., 2006 a, Biomarcadores etológicos no invasivos de estrés ambiental: estudio comparativo en dos teleósteos de ecosistemas de la región pampeana argentina. Revista de Toxicología (España), 23: 11-16.

EISSA, B.L., SALIBIÁN, A. \& FERRARI, L., 2006 b, Behavioral alterations in juvenile Cyprinus carpio exposed to sublethal waterborne cadmium. Bull. Environ. Cont. Toxicol., 77: 931937. http://dx.doi.org/10.1007/s00128-006-1233-2

ELLMAN, G.L., COURTNEY, K.D., ANDRES, JR. V. \& FEATHERSTONE, R.M., 1961, A new and rapid colorimetric determination of acetylcholinesterase activity. Biochem. Pharmacol., 7: 88-95 http://dx.doi.org/10.1016/00062952(61)90145-9

EVANS, D.H., PIERMARINI, P.M., CHOE, K.P., 2005, The multifunctional fish gill: dominant site of gas exchange, osmoregulation, acid-base regulation, and excretion of nitrogeneous waste. Physiol. Revs., 85: 97-177. http://dx.doi. org/10.1152/physrev.00050.2003

FERRARI, L., EISSA, B.L., OSSANA, N.A. \& SALIBIÁN, A., 2009, Effects of sublethal cadmium on gills in three teleosteans species: scanning electron microscope study. Int. J. Environ. Health, 3: 410-426. http:// dx.doi.org/10.1504/IJENVH.2009.030111

GERHARDT, A., JANSSENS DE BISTHOVEN, L., MO, Z., WANG, C., YANG, M. \& WANG, Z., 2002, Short-term responses of Oryzias latipes (Pisces: Adrianichthydae) and Macrobrachium nipponense (Crustacea: Palaemonidae) to municipal and pharmaceutical waste water in Beijing, China: survival, behaviour, biochemical biomarkers. Chemosphere, 47: 35-47. http://dx.doi.org/10.1016/S0045-6535(01)00223-5

GIORGI, A., 2000, Costo de la contaminación del Río Luján. UNLu Ciencia 2: 42-47. 
GROTHE, D.R., DICKSON, K.L. \& REED-JUDKINS, D.K. (eds.), 1996, Whole effluent toxicity testing: an evaluation of methods and prediction of receiving system impacts. SETAC Special Publication, SETAC Press, Pensacola, FL, 340 pp.

LEY NACIONAL DE RESIDUOS PELIGROSOS 24051 (www. ambiente.gov.ar, visitado noviembre 2012).

LIU, J., QU, W. \& KADIISKA, M.B., 2009, Role of oxidative stress in cadmium toxicity and carcinogenesis. Toxicol. Appl. Pharmacol., 238: 209-214. http://dx.doi.org/10.1016/j.taap.2009.01.029.

LOWRY, O.H., ROSEBROUGH, N.J., FARR, A.L. \& RANDALL, R.J., 1951, Protein measurements with Folin phenol reagent. $J$. Biol. Chem., 193: 265-275.

MORAES, B.S., LORO, V.L., GLUSCZAK, L., MENEZES, C., MARCHEZAN, E \& MACHADO, S.O. (2007) Effects of four rice herbicides on some metabolic and toxocology parameters of teleost fish (Leporinus obtusidens). Chemosphere, 68:15971601. http://dx.doi.org/10.1016/j.chemosphere.2007.03.006

OSSANA, N.A., CASTAÑÉ, P.M., SARMIENTO \& P.L., SALIBIÁN, A. (2010) Do Lithobates catesbeianus tadpoles acclimatise to sub-lethal copper? Int. J Environ Health, 4: 342354. http://dx.doi.org/10.1504/IJENVH.2010.037499

OSSANA N.A. (2011) Biomarcadores de contaminación acuática: estudios de los río Luján y Reconquista. Tesis doctoral, Facultad de Ciencias Exactas y Naturales, Universidad de Buenos Aires.
RODRIGUEZ KAIZER, R., LORO, V.L. \& SCHETINGER, M.R.C., 2009, NTPDase and acetylcholinesterase activities in silver catfish, Rhamdia quelen (Quoy \& Gaimard, 1824) (Heptapteridae) exposed to interaction of oxygen and ammonia levels. Neotrop. Ichthyol., 7: 635-640.

SÁNCHEZ CARO, A. (2010). Calidad del agua del río Luján. En Carballo, C. T. (Ed.), Información ambiental de la Cuenca del rio Luján: aportes para la gestión integral del agua (pp. 123131) Buenos Aires: Prometeo Libros.

SIEGEL, S., CASTELLAN, N.J., 1995, Estadística no paramétrica aplicada a las ciencias de la Conducta. Editorial Trillas. Mexico, $437 \mathrm{pp}$.

SLOOF, W., VAN KREIJL, C.F. \& BAARS, A.J., 1983, Relative liver weights and xenobiotic-metabolizing enzymes of fish from polluted surface waters in the Netherlands. Aquat. Toxicol., 4: 1-14. http://dx.doi.org/10.1016/0166-445X(83)90057-7

THOPHON, S., KRUATRACHUE, M., UPATHAM, E.S., POKETHITIYOOK, P., SAHAPHONG, S. \& JARITKHUAN, S., 2003, Histopathological alterations of white seabass, Lates calcarifer, in acute and subchronic cadmium exposure. Environ. Pollut., 121: 307-320. http:// dx.doi.org/10.1016/S02697491(02)00270-1

ZAR, J. H., 2010, Biostatistical Analysis, New Jersey: Pearson Prentice Hall. 